\title{
Una experiencia que aporta al desarrollo integral y al pensamiento matemático de niños de 5 y 6 años
}

Volumen 6 N. ${ }^{\circ} 48$ enero-junio de 2020

ISSN: 0122-4328

ISSN-E: 2619-6069 pp. $117-128$
An Experience that Contributes to the Integral Development and Mathematical Thinking of 5 and 6 Year-Old Children
Uma experiência que contribui para o desenvolvimento integral e o pensamento matemático de crianças de 5 e 6 anos

Kelly Johana Duque Gutiérrez*

Lyda Constanza Mora Mendieta**

Fecha de recepción: 30-01-20

Fecha de aprobación: 27-04-20

\section{PARA CITAR ESTE ARTÍCULO}

Duque Gutiérrez, K. y Mora Mendieta, L. (2020). Una experiencia que aporta al desarrollo integral y al pensamiento matemático de niños de 5 y 6 años. Nodos y Nudos, 6(48). https://doi.org/ 10.17227/nyn.vol6.num48-11271 


\section{RESUMEN}

Este artículo de investigación, resultado del trabajo de grado "Tareas matemáticas para potenciar el desarrollo integral del niño en preescolar" (Duque, 2018), presenta una experiencia de aula alrededor de tareas matemáticas centradas en el pensamiento matemático, por medio de la cual se buscó potenciar el desarrollo integral de niños de cinco y seis años, identificando las dimensiones (biológica, social y cognitiva) a las que aportaban tales tareas. Para ello, luego de la identificación del contexto y la elaboración de un marco de referencia, se seleccionaron o diseñaron tareas matemáticas. Posteriormente se llevaron al aula y se registraron los procesos y dimensiones evidenciados por los niños.

Volumen 6 N.o 48 enero-junio de 2020 ISSN: 0122-4328 ISSN-E: 2619-6069 pp. $117-128$
El principal resultado de la experiencia es que el desarrollo del pensamiento matemático es posible desde las primeras edades escolares y que estas también contribuyen al desarrollo integral de los niños, siempre que su planeación se haga con tal intencionalidad.

Palabras clave: proceso cognitivo; educación de la primera infancia; pensamiento; matemáticas

\section{ABSTRACT}

This research article, result of the degree work "Mathematical tasks to enhance the integral development of the child in preschool" (Duque, 2018), presents a classroom experience around mathematical tasks focused on mathematical thinking, through which it sought to promote the integral development of five and six-year-old children, identifying the dimensions (biological, social and cognitive) to which such tasks contributed. For this, after the identification of the context and the elaboration of a frame of reference, mathematical tasks were selected or designed. Later they were taken to the classroom and the processes and dimensions evidenced by the children were recorded. The main result of the experience is that the development of mathematical thinking is possible from the first school ages and that these also contribute to the integral development of children, since their planning is done with such intentionality.

Keywords: cognitive process; early childhood education; thinking, mathematics

\section{RESUMO}

Este artigo de investigação, resultado do trabalho de licenciatura "Tarefas matemáticas para melhorar o desenvolvimento integral da criança na pré-escola" (Duque, 2018), apresenta uma experiência de sala de aula em torno de tarefas matemáticas centradas no pensamento matemático, através das quais buscou promover o desenvolvimento integral de crianças de cinco e seis anos, identificando as dimensões (biológicas, sociais e cognitivas) para as quais contribuiam tais tarefas. Para isso, após a identificação do contexto e a elaboração de um referencial básico, foram selecionadas ou elaboradas tarefas matemáticas. Posteriormente levadas para a sala de aula e registrados os processos e dimensões evidenciados pelas crianças. 0 principal resultado da experiência é que o desenvolvimento do pensamento matemático é possivel desde as primeiras idades escolares e que estas também contribuem para o desenvolvimento integral das crianças, desde que seu planejamento seja feito com essa intencionalidade.

Palavras-chave: processo cognitivo; educação infantil; pensamento; matemática 


\section{Introducción y contextualización}

Las tareas matemáticas, entendidas como aquellas que tienen el objetivo principal de desarrollar un proceso matemático (Chamorro, 2003), son poco usuales en el ámbito escolar cercano a las autoras de este documento, en tanto lo frecuente es que el centro sean los conceptos o los algoritmos (conocidos como temas: los números, las operaciones, las figuras geométricas, entro otros) a pesar de las múltiples voces que proponen que el pensamiento matemático sea el centro del proceso de formación en el ámbito escolar (Ordoñez, 2009). Así que en el 2017, se decidió idear un proyecto de práctica pedagógica en la Licenciatura en Matemáticas de la Universidad Pedagógica Nacional (UPN) desde una mirada investigativa inicial que posibilitara llevar tareas matemáticas a los más pequeños, las cuales, a su vez, aportaran a su desarrollo integral. Al ser popular la creencia de que "las matemáticas solo contribuyen al desarrollo cognitivo" surge la pregunta sobre si pueden las tareas matemáticas contribuir al despliegue de varias dimensiones del proceso formativo de los niños. Emprendida esta iniciativa el Instituto Pedagógico Nacional (IPN), escuela de práctica investigativa e innovadora de la UPN, decidió participar en este proyecto y fue así como en el 2018 se llevó a cabo con los niños de uno de los cursos de Transición (conformado por veintitrés niños de 5 y 6 años) gracias al apoyo institucional, la profesora Martha Jaimes y de los padres de familia, quienes dieron su aval para la grabación de cada una de las sesiones de clase.

Las tareas implicaron que los niños y niñas jugaran, interactuaran con sus compañeros e hicieran uso de sus conocimientos, así como que desarrollaran su pensamiento matemático y dimensiones del desarrollo integral.

Se organizaron diez tareas relacionadas con el proyecto de aula denominado "Los piratas". Terminada cada sesión de clase se analizaba el alcance de esta y finalizado el periodo de intervención se valoraron los logros alcanzados, para ello se diseñaron instrumentos que permitieron realizar el análisis y el planteamiento de algunas conclusiones parciales sobre los procesos y las dimensiones evidenciadas en la puesta en práctica de las tareas respecto a lo planeado.

\section{Marco de referencia}

Para llevar a cabo este proyecto de investigación se requirió precisar dos elementos fundamentales: desarrollo integral y pensamiento matemático.

\section{Desarrollo integral}

La educación, y en particular la educación inicial, debe velar por el desarrollo integral del niño y así lo piensan distintas entidades. En el contexto internacional, el Estado Mundial de la Infancia del 2001 considera que los programas de la primera infancia deben desarrollar en el niño sus capacidades cognitivas, emocionales, sociales y físicas (Unicef, 2001). En el contexto nacional se encuentran diversos organismos que exponen en sus documentos su postura con relación a lo que significa el desarrollo en la primera infancia:

» La República de Colombia, por medio de la Ley 115 (1994), se refiere a un conjunto de procesos: biológico, cognoscitivo, sicomotriz, socio-afectivo y espiritual.

»El Ministerio de Educación Nacional, a través de los Lineamientos para Preescolar (1998), fija su atención en un conjunto de dimensiones: socio-afectiva, corporal, cognitiva, comunicativa, estética, espiritual y ética.

» La Secretaría de Educación Distrital, en el Lineamiento Pedagógico y Curricular (2013), entiende el desarrollo humano como un todo compuesto por las dimensiones personal-social, corporal, comunicativa, artística y cognitiva.

Buscando establecer la menor cantidad de dimensiones, por efecto de sintesis, y atendiendo a que las diferentes dimensiones se relacionan entre sí, se decidió tener en cuenta tres: cognitiva (que incluye las dimensiones cognitiva y comunicativa), social (donde se incluyen: dimensión social, personal, ética, emocional, afectiva, espiritual, estética y artística) y biológica (conformada por las dimensiones física, corporal y psicomotriz). 


\section{Pensamiento matemático}

El pensamiento matemático se entiende como un conjunto de actividades intelectuales internas (por ejemplo abstracción, justificación, visualización, estimación o razonamiento por hipótesis, modelación y comparación) que se manifiestan en situaciones de resolución de problemas, toma de una decisión o extracción de una conclusión.

\section{Procesos del pensamiento matemático}

Puesto que la actividad matemática no se concreta en sus contenidos sino en sus procesos, ser matemáticamente competente requiere habilidades en los siguientes procesos generales del pensamiento matemático (García y Salazar, 2009; MEN, 1998; 2006): resolución de problemas, razonamiento, comunicación, modelación y elaboración, comparación y ejercitación de procedimientos.

Por su parte el National Council of Teachers of Mathematics (2000) propone que para lograr una sociedad que tenga la capacidad de pensar y razonar matemáticamente se requiere trabajar alrededor de: resolución de problemas, razonamiento y demostración, comunicación, hacer conexiones y representación.

El Programme for International Student Assessment (2012) también considera algunos procesos como capacidades fundamentales sobre las cuales plantea las evaluaciones que lidera a nivel internacional, estas son: formulación matemática de situaciones; empleo de conceptos, datos y procedimientos y razonamientos matemáticos e interpretación; aplicación y valoración de los resultados matemáticos.

Resultan evidentes las relaciones entre lo propuesto por entidades nacionales e internacionales, y establecidas tales conexiones se eligieron los siguientes procesos que fueron la base del diseño de las tareas que se llevaron a cabo: resolver problemas, razonar y argumentar, comunicar, hacer conexiones, representar y generalizar.

\section{Metodología}

Como se mencionó en la primera parte de este documento, la experiencia de investigación formativa que se presenta nació de un proyecto de práctica pedagógica, en el marco de la Licenciatura en Matemáticas de la upN en el 2017 y puesta en marcha en el IPN durante el primer semestre del 2018 con estudiantes de Transición, cuyas edades oscilaban entre los 5 y 6 años.

Las tareas matemáticas se diseñaban semanalmente determinando con anterioridad los alcances esperados. La tabla 1 resume los títulos de las diez tareas presentadas, y las dimensiones y procesos que se buscaba potenciar:

Tabla 1. Tareas propuestas, dimensiones y procesos que se deseaba potenciar

\begin{tabular}{|c|c|c|c|c|c|c|c|c|c|c|}
\hline & & Dime & & & Pensar & nto i & mát & & & \\
\hline Tareas $^{12}$ & Breve descripción & 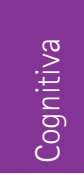 & $\begin{array}{l}\overline{\frac{\pi}{L}} \\
\stackrel{0}{n}\end{array}$ & $\begin{array}{l}\frac{\pi}{9} \\
\frac{.0}{\circ} \\
\frac{0}{0}\end{array}$ & 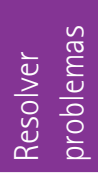 & 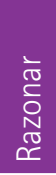 & . & 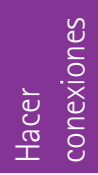 & 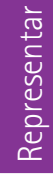 & 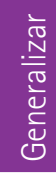 \\
\hline 1. Tesoro & $\begin{array}{l}\text { Describir el contenido } \\
\text { de un tesoro (caja con } \\
\text { algunos juguetes) por } \\
\text { medio de dibujos }\end{array}$ & $X$ & $x$ & $x$ & & $X$ & $X$ & & $X$ & \\
\hline 2. Mapa & $\begin{array}{l}\text { Describir la ubicación de } \\
\text { un tesoro usando una } \\
\text { cuadrícula }\end{array}$ & $x$ & & $x$ & $X$ & & $X$ & & $X$ & \\
\hline
\end{tabular}

1 Las tareas llevadas a cabo se encuentran en el repositorio de tesis y trabajos de grado de la Universidad Pedagógica Nacional: http://repositorio.pedagogica.edu.co/ handle/20.500.12209/9528

2 Las tareas 1 a 4 están inspiradas en algunas presentadas en Didáctica de las matemáticas (Chamorro, 2005). 


\begin{tabular}{|c|c|c|c|c|c|c|c|c|c|c|}
\hline & & Dime & & & Pensar & nto & mát & & & \\
\hline Tareas $^{12}$ & Breve descripción & 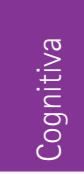 & $\begin{array}{l}\overline{\frac{\pi}{L}} \\
\text { D }\end{array}$ & $\begin{array}{l}\frac{\pi}{5} \\
\frac{0}{0} \\
\frac{0}{0}\end{array}$ & 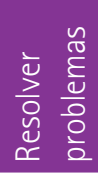 & 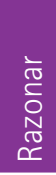 & 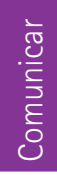 & 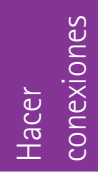 & 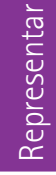 & 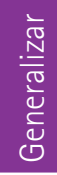 \\
\hline 3. Barco & $\begin{array}{l}\text { Solicitar por escrito } \\
\text { al docente las fichas } \\
\text { de colores necesarias } \\
\text { para replicar un barco } \\
\text { construido en una hoja }\end{array}$ & $x$ & & $x$ & $x$ & $x$ & $x$ & & $x$ & \\
\hline $\begin{array}{l}4 . \\
\text { Indicaciones } \\
\text { al tesoro }\end{array}$ & $\begin{array}{l}\text { Guiar a un compañero } \\
\text { hacia un tesoro escondido }\end{array}$ & $x$ & & $x$ & & & $x$ & $x$ & $x$ & \\
\hline $\begin{array}{l}\text { 5. Tipos de } \\
\text { barcos }\end{array}$ & $\begin{array}{l}\text { Replicar con bloques de } \\
\text { Dienes barcos presentados } \\
\text { por la profesora }\end{array}$ & $x$ & & & & $x$ & & & $x$ & \\
\hline $\begin{array}{l}\text { 6. Completar } \\
\text { el mapa }\end{array}$ & $\begin{array}{l}\text { Completar un mapa que } \\
\text { solo contiene el recorrido, } \\
\text { sin referencias }\end{array}$ & $x$ & $x$ & $x$ & $x$ & $x$ & & $x$ & $x$ & \\
\hline 7. Piratas & $\begin{array}{l}\text { Elaborar un sombrero } \\
\text { doblando papel y } \\
\text { decorarlo }\end{array}$ & $x$ & & $x$ & $x$ & & $x$ & $x$ & $x$ & \\
\hline 8. Contar & $\begin{array}{l}\text { Interpretar las diversas } \\
\text { representaciones de } \\
\text { números en tarjetas }\end{array}$ & $x$ & & & & $x$ & & & $x$ & \\
\hline 9. Rondas & $\begin{array}{l}\text { Patrones corporales en } \\
\text { canciones de piratas }\end{array}$ & $x$ & $x$ & $x$ & $x$ & & $x$ & & $x$ & $x$ \\
\hline 10. Navegar & $\begin{array}{l}\text { Patrones gráficos para } \\
\text { predecir el tiempo al salir } \\
\text { a navegar }\end{array}$ & $x$ & & $x$ & $x$ & $x$ & & & $x$ & $x$ \\
\hline
\end{tabular}

Fuente: elaboración propia.

Esta tabla hizo evidente que en efecto se estaba apuntando a todas las dimensiones del desarroIlo integral y procesos del pensamiento matemático considerados. Antes de cada una de las sesiones se realizó una planeación que posteriormente fue modificada, de acuerdo con lo sucedido durante la clase.

Cada planeación de clase contaba con los siguientes elementos: objetivo de enseñanza, dimensiones y procesos que deseaban potenciary desarrollo de la sesión.

Una vez realizadas las intervenciones en el aula se hacia una breve narración de las situaciones presentadas en la clase que tuvieran relación con las dimensiones del desarrollo integral del niño (DDI) y los procesos del pensamiento matemático (PPM). Esta narración se organizaba en el formato "Alcance de la actividad" que contaba con los siguientes componentes:

Dimensiones del desarrollo integral: en esta sección se incluian las dimensiones que estuvieron presentes durante la sesión, esto acompañado de soportes (como relatos de lo ocurrido y fotografías del trabajo producido).

Procesos del pensamiento matemático: en esta sección se ubicaban los procesos que estuvieron presentes durante la sesión, esto acompañado de soportes (como relatos de lo ocurrido y fotografías del trabajo producido). 
Consideraciones para la siguiente sesión: usando los eventos ocurridos en la clase se presentaban los elementos que se debían contemplar en una próxima puesta en acción.

Con el fin de dar una mirada más sistemática a las sesiones de clase, se establecieron algunos indicadores de logro tanto para las dimensiones como para los procesos, como se muestra enseguida.

\section{Indicadores para las dimensiones}

\section{Desarrollo cognitivo:}

1. Representa el mundo real a través de símbolos (dibujos, objetos, imágenes, entre otros)

2. Responde y hace preguntas como: ¿Por qué? ¿Cómo? ¿Para qué?

3. Propone problemas o soluciones a estos

4. Logra expresar sus ideas a través del algún tipo de comunicación

5. Comprende relaciones como más que, menos que, igual a, a la izquierda de, a la derecha de, delante de y atrás de

\section{Desarrollo social:}

1. Expresa acuerdo o desacuerdo con actividades propuestas

2. Establece relaciones asertivas con las personas que lo rodean

3. Reconoce lo que puede o no hacer en determinados momentos (lo que está bien y lo que no está bien)

4. Expresa sus emociones

\section{Desarrollo biológico:}

1. Representa lo que observa e interpreta por medio de dibujos y su propia grafía ${ }^{3}$

2. Representa lo que observa e interpreta por medio de su expresión corporal ${ }^{4}$
Indicadores para los procesos del desarrollo del pensamiento matemático:

Resolver problemas:

1. Se hace preguntas (o formula inquietudes) y propone problemas

2. Comprende el/los problemas propuestos

3. Planifica cómo resolver un problema dado

4. Explica el proceso realizado al resolver un problema

5. Valora la(s) solución(es) dada(s) a un problema

\section{Razonar y argumentar:}

1. Establece propiedades y relaciones, las comprueba de algún modo

2. Se hace y responde preguntas como ¿Por qué? ¿Cómo? ¿Esto siempre es así? ¿Y si cambiamos esto qué pasa?, entre otras

3. Hace demostraciones empíricas

4. Hace uso de frases donde utiliza conectores como $y, 0$, si, todos, algunos, entonces y porque

\section{Comunicar:}

1. Expresa ideas de manera oral, escrita, o gestual

2. Comprende, interpreta y evalúa ideas

3. Escucha las ideas de las personas que lo rodean

\section{Hacer conexiones:}

1. Relaciona los nuevos aprendizajes con sus intereses

2. Relaciona lo que está sucediendo/aprendiendo con su(s) experiencia(s)/conocimiento(s) previo(s)

3. Relaciona sus nuevos aprendizajes con el mundo real

\section{Representar:}

1. Hace traducciones entre representaciones mentales y representaciones externas

2. Utiliza y lee distintos medios para hacer representaciones (enactivos, icónicos, simbólicos)

\section{Generalizar:}

1. Identifica el patrón y lo sigue

2. Expresa o describe el patrón o secuencia hallada

3. Representa el patrón hallado

4. Justifica el patrón o la secuencia establecida

3 Adaptado Derechos Básicos de Aprendizaje, MEN, 2017. 
Los números asignados anteriormente se usaron para registrar en la tabla 2 los indicadores observados en los niños en cada una de las tareas. La tabla consta de dos entradas: en la primera columna se registraron los nombres de los estudiantes (que se leen como E1, $E 2, \ldots$, E23) y en la parte superior para cada dimensión y proceso del pensamiento matemático se listan las diez tareas diseñadas $(A 1, A 2, \ldots, A 10)$.

Tabla 2. Registro del indicador evidenciado en cada tarea

\begin{tabular}{|c|c|c|c|c|c|c|c|c|c|c|c|c|c|c|c|c|c|c|c|c|c|c|c|c|c|c|c|c|c|c|}
\hline & & & & & ogniti & & & & & & & & & & & Soci & & & & & & & & & & Bioló & gico & & & \\
\hline Niño & A1 & A2 & A3 & \begin{tabular}{|l|l} 
A4 \\
\end{tabular} & A5 & A6 & A7 & $A 8$ & $\mathrm{~A} S$ & & A10 & A1 & A2 & A3 & A4 & \begin{tabular}{|l|l|} 
A \\
\end{tabular} & A6 & A7 & AB & A9 & A10 & A1 & A2 2 & A3 & A4 & \begin{tabular}{|l|l|} 
A \\
\end{tabular} & \begin{tabular}{|l|l} 
A6 \\
\end{tabular} & \begin{tabular}{l|l}
$A 7$ & $A$ \\
\end{tabular} & \begin{tabular}{l|l|}
8 & A9
\end{tabular} & A10 \\
\hline$E 1$ & \begin{tabular}{|l|}
1,2 \\
\end{tabular} & 1 & & \begin{tabular}{|l|}
5 \\
\end{tabular} & 1 & & 4 & 2 & & 5 & & 3 & & \begin{tabular}{|l|}
3 \\
\end{tabular} & & & & & & 2 & & & & & & & & & \begin{tabular}{l|l|} 
& 2 \\
\end{tabular} & \\
\hline E2 & 1,2 & 1 & $1,4,5$ & 5 & 1 & 2 & 4 & 2 & & 5 & 2 & 3 & & 3 & & & 2 & & & 2 & & & & 1 & & & 2 & 1 & 2 & \\
\hline $\mathrm{E} 3$ & 1,2 & 1 & 1,4 & 5 & 1 & & 4 & 2 & & 5 & 2 & 3 & & 3 & & & & & & 2 & & & & 1 & & & 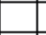 & 1 & 2 & 1 \\
\hline$E 4$ & 1,2 & 1 & 1,4 & 5 & 1,2 & 2 & 4 & 2 & & 5 & 2 & 3 & & 3 & & & 2 & & & 2 & & & 1 & 1 & & & 2 & 1 & 2 & 1 \\
\hline E5 & 1,2 & 1 & 1,4 & 5 & 1 & 2 & 4 & 2 & & 5 & & 3 & & 3 & & & 2 & & & 2 & & & & 1 & 2 & & 2 & & 2 & 1 \\
\hline$E 6$ & 1,2 & 1 & & 5 & 1 & & 4 & 2 & & 5 & & 3 & & & & & 2 & & & 2 & & & & 1 & & & 2 & 1 & 2 & \\
\hline$E 7$ & 1,2 & 1 & 1,4 & 5 & 1 & 2 & 4 & 2 & & 5 & 2 & 3 & & 3 & & & 2 & & & 2 & & & & 1 & 2 & & 2 & 1 & 2 & \\
\hline$E 8$ & 1,2 & 1 & 1,4 & 5 & 1 & 2 & 4 & & & 5 & 2 & 3 & & 3 & & & 2 & & & 2 & & & & & & & 2 & & 2 & \\
\hline E9 & 1,2 & 1 & & 5 & 1 & 2 & 4 & 2 & 2 & 5 & & 3 & & 3 & & & 2 & & & 2 & & & 1 & & & & 2 & 1 & 2 & \\
\hline$E 10$ & 1,2 & 1 & 1,4 & 5 & 1 & 2 & 4 & 2 & & 5 & 2 & 3 & & 3 & & & 2 & & & 2 & & 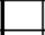 & & 1 & & & 2 & 1 & 2 & \\
\hline E11 & 1,2 & 1 & 1,4 & 5 & 1 & 2 & 4 & 2 & 2 & 5 & 2 & 3 & & 3 & & & 2 & & & 2 & & 1 & 1 & 1 & & & 2 & 1 & 2 & 1 \\
\hline$E 12$ & 1,2 & 1 & 1,4 & 5 & 1 & 2 & 4 & 2 & & 5 & 2 & 3 & & 3 & & & 2 & & & 2 & & 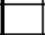 & 1 & 1 & & & 2 & 1 & 2 & 1 \\
\hline E13 & 1,2 & 1 & 1,4 & 5 & 1 & 2 & 4 & 2 & & 5 & 2 & 3 & & 3 & & & 2 & & & 2 & & & 1 & 1 & & & 2 & 1 & 2 & 1 \\
\hline E14 & 1,2 & 1 & 1,4 & 5 & 1 & & 4 & 2 & 2 & 5 & & 3 & & 3 & & & & & & 2 & & & & & & & & & 2 & \\
\hline E15 & 1,2 & 1 & 1,4 & 5 & 1 & & 4 & 2 & & 5 & & 3 & & 3 & & & 2 & & & 2 & & 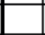 & & 1 & & & 2 & 1 & 2 & 1 \\
\hline$E 16$ & 1,2 & 1 & 1,4 & 5 & 1 & 2 & 4 & 2 & & 5 & & 3 & & 3 & & & 2 & & & 2 & & 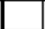 & & 1 & & & 2 & 1 & 2 & 1 \\
\hline$E 17$ & $1,2,4$ & 1 & 1,4 & 5 & 1 & 2 & 4 & 2 & 2 & 5 & 2 & 3 & & 3 & & & 2 & & & 2 & & 1 & 1 & 1 & & & 2 & 1 & 2 & \\
\hline$E 18$ & $1,2,4$ & 1 & 1,4 & 5,4 & 1 & 2 & 4 & 2 & 2 & 5 & 2 & 3 & & 3 & & & 2 & & & 2 & & 1 & 1 & 1 & & & 2 & 1 & 2 & 1 \\
\hline$E 19$ & & 1 & 1,4 & 5 & 1 & & 4 & 2 & 2 & 5 & & & & 3 & & & 2 & & & 2 & & & & 1 & & & 2 & 1 & 2 & 1 \\
\hline E20 & 1,2 & 1 & 1,4 & 5 & 1 & 2 & 4 & 2 & 2 & 5 & 2 & 3 & & 3 & & & 2 & & & 2 & & 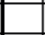 & & 1 & & & 2 & 1 & 2 & 1 \\
\hline E21 & $1,2,4$ & 1 & 1,4 & 5 & 1 & 2 & 4 & 2 & & 5 & 2 & 3 & & 3 & & & 2 & & & 2 & & 1 & 1 & 1 & & & 2 & 1 & 2 & 1 \\
\hline$E 22$ & \begin{tabular}{|l|}
1,2 \\
\end{tabular} & 1 & 1,4 & 5 & 1 & 2 & 4 & 2 & & 5 & & 3 & & 3 & & & 2 & & & 2 & & & 1 & 1 & & & 2 & 1 & 2 & \\
\hline
\end{tabular}

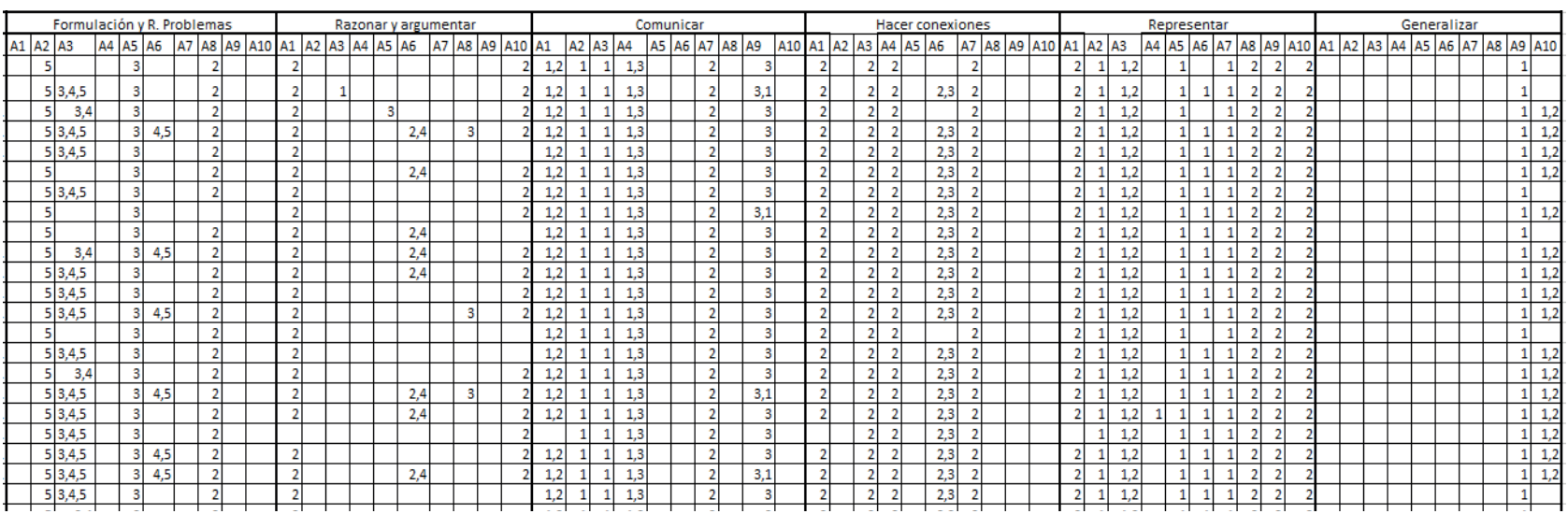

Fuente: elaboración propia.

\section{Resultados}

De acuerdo con el objetivo propuesto se hicieron dos tipos de análisis. En primer lugar, se analizó cada una de las tareas puesta en escena con el fin de reportar si hubo evidencia de las dimensiones o procesos propuestos, para ello se usó el formato "Alcance de la actividad"; además, de acuerdo con la información registrada en la tabla de análisis general, se elaboró un gráfico sencillo de barras que permitió consolidar los hallazgos para cada tarea.

Posteriormente, se hizo un análisis por cada una de las dimensiones y procesos por medio de gráficos que se alimentaron de la información registrada en la tabla de análisis general, donde se consignaron los indicadores alcanzados. 
Enseguida se presenta un ejemplo de las tareas realizadas (tarea 10), inspirada en la rutina que tenían los estudiantes de registrar el clima en un calendario de su salón de clase. Luego se muestra el primer tipo de reflexión sobre cómo se analizó una de las dimensiones y uno de los procesos.

\section{Análisis por tareas}

Tarea 10: ¡A navegar!

Objetivo de enseñanza: Fortalecer la identificación y seguimiento de patrones.

Dimensiones que desea potenciar: cognitiva (el niño debe argumentar el porqué de sus respuestas) y biológica (los niños representan sus interpretaciones por medio de dibujos).

Procesos del pensamiento matemático que desea potenciar: argumentación (el niño responde a preguntas como ¿Por qué? ¿Cómo?), representación (se deben idear medios para representar el patrón que se va a inventar) y generalización (el niño identifica el patrón y se espera que lo siga).

Desarrollo de la sesión: se mostró el calendario del mes de abril (Véase figura 1) y se contó a los niños que "un pirata meteorólogo me ha contado que el estado del tiempo para este mes va a ser así:

\begin{tabular}{|l|l|l|l|l|l|l|}
\hline \multicolumn{7}{|c|}{ Abril 2018 } \\
\hline Domingo & Lunes & Martes & Miércoles & Jueves & Viernes & Sábado \\
\hline 1 & 2 & 3 & 4 & 5 & 6 & 7 \\
\hline 8 & 9 & 10 & 11 & 12 & 13 & 14 \\
\hline 15 & 16 & 17 & 18 & 19 & 20 & 21 \\
\hline 22 & 23 & 24 & 25 & 26 & 27 & 28 \\
\hline 29 & 30 & & & & & \\
\hline
\end{tabular}

Figura 1. Estado del tiempo para el mes de abril

Fuente: elaboración propia.

Para el primer día será lluvioso, para el segundo día soleado, para el tercer día lluvioso y así continuará durante todo el mes". Con esta aclaración se entregó a los niños el calendario y pegatinas de nubes y soles, continuamos "Si solo podemos salir a navegar en días soleados, ¿podríamos salir a navegar el 12 de abril? ¿Y podríamos salir el 26? ¿Y el 30?
¿Los lunes podremos salir? ¿Y los sábados? ¿Cuál sería la secuencia del estado del tiempo que hay para este mes? ¿Cómo la escribirían?".

El calendario se convirtió en un medio para que los estudiantes justificaran sus respuestas, puesto que, al hacer preguntas como "¿El 12 de abril podremos salir a navegar?" los estudiantes miraron su hoja y respondieron "si"; al preguntar por qué afirmaron contestaron "porque así lo pusimos en el calendario". Esto hizo evidente la capacidad de los niños para responder a preguntas de tipo argumentativo y valerse de algún medio para justificar.

Se pidió a los niños que representaran la secuencia para el estado del tiempo en el mes de mayo y se presentaron las siguientes situaciones:

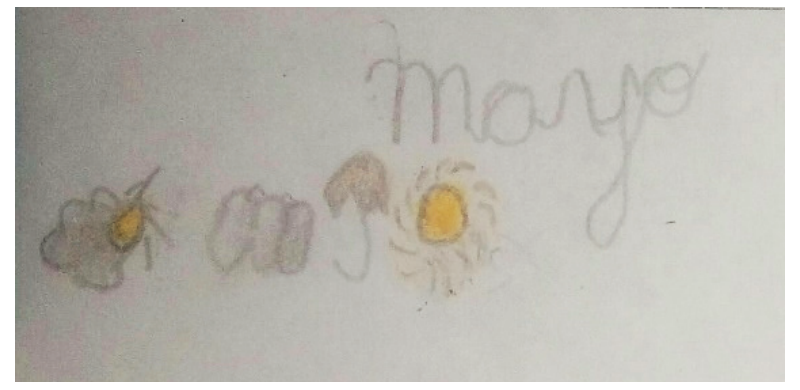

Figura 2. Núcleo ABCD inventado por E

E8 inventó una secuencia figurativa (esto por el tipo de tarea que se pedía) que tiene un núcleo $A B C D$ (Véase figura 2), la cual resulta ser más compleja que las secuencias corporales y figurativas que se habian trabajado hasta el momento.

Un grupo de estudiantes hace uso del núcleo $A B$ valiéndose de otros elementos, es decir, estados del tiempo diferentes a los nombrados recientemente (Véase figura 3).

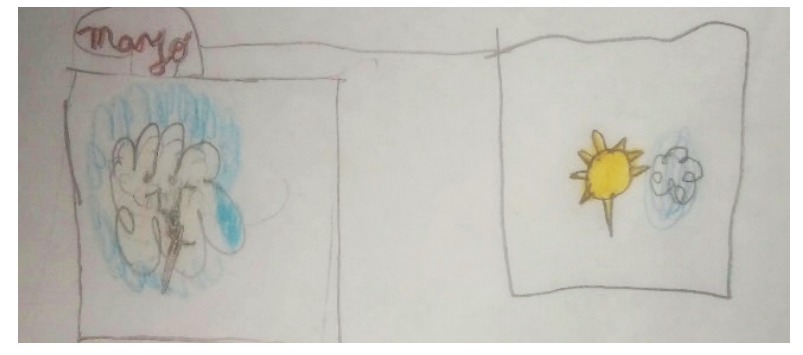

Figura 3. Núcleo AB inventado por 8 niños 
Otro grupo de estudiantes consideraba que no basta con representar el núcleo, vieron la necesidad de repetir el patrón para dar a entender que este se repetiria (Véase figura 4).

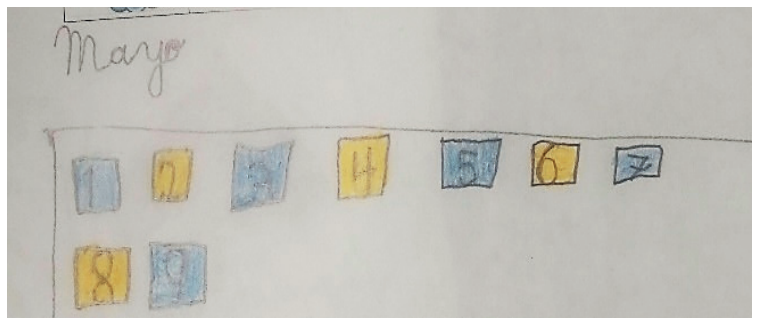

Figura 4. Núcleo AB

Al preguntar a E2 "¿Qué significa su representación (Véase figura 5)?" respondió que "el 1 va a ser soleado y el dos también y el 3 también y así todo el mes", este estudiante inventa un patrón de núcleo A.

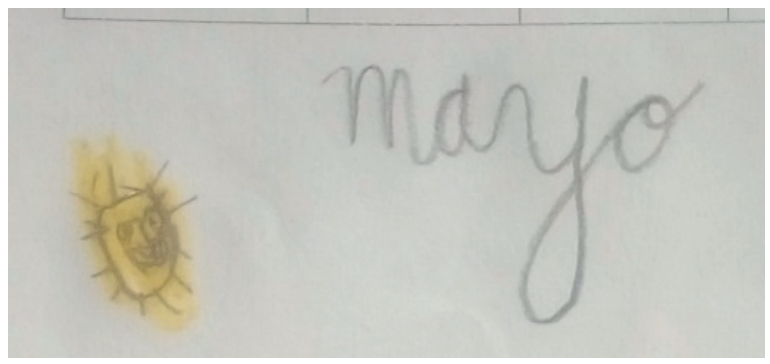

Figura 5. Núcleo A inventado por E2

Decir a los niños que la secuencia para el mes de abril sería nublado-soleado bastó para que completaran su calendario con pegatinas de manera adecuada, por lo que es posible decir que los niños siguen patrones (indicador Generalización 1). Sin embargo, solo 17 estudiantes expresaron una secuencia (indicador Generalización 2) dado que los 6 estudiantes restantes dibujaron barcos y situaciones relacionadas con los piratas, dejando de lado la tarea solicitada.

\section{Análisis por dimensiones y procesos del pensamiento matemático}

Aunque este análisis se realizó de manera disjunta por fines analíticos, se reconoce la relación de las dimensiones entre sí, de los procesos entre sí y en algunas ocasiones entre dimensiones y procesos. Para realizar el análisis por dimensiones/procesos se construyó una gráfica que relacionaba la tarea correspondiente con la cantidad de niños que revelaron la presencia de la dimensión o proceso que se estaba analizando. Si una de las tareas hechas no se relaciona en determinada gráfica esto indica que dicha dimensión/proceso no se evidenció en la tarea.

\section{Dimensión biológica}

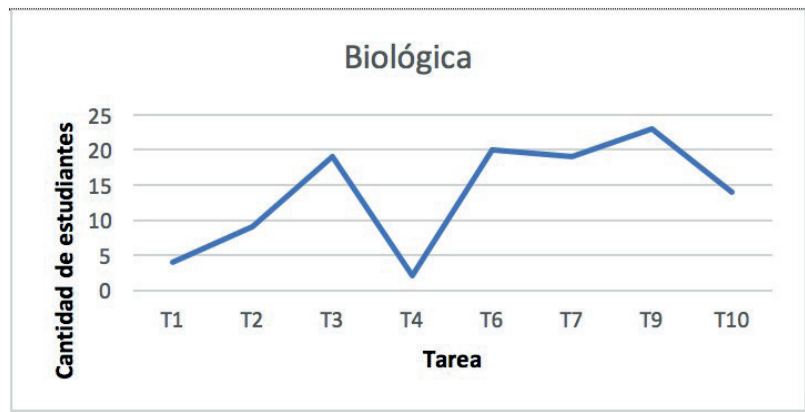

Figura 6. Alcance de la dimensión biológica en la realización de las tareas

Fuente: elaboración propia.

La presencia de la dimensión biológica durante las tareas realizadas no fue constante, sin embargo tuvo un alto índice, pues de las 10 tareas propuestas 8 aportaron al desarrollo de esta dimensión. Adicionalmente, en 5 de estas 8 tareas más del $50 \%$ de los niños presentaron actitudes que mostraban que la clase estaba poniendo en juego su dimensión biológica (Véase figura 6).

En relación con dicha dimensión, las tareas estuvieron centradas en la representación de ideas por medio de dibujos, grafías y expresión corporal, aspectos que no estuvieron presentes en las tareas 1 y 4 donde se presentó el índice más bajo dado que los niños no coloreaban o no escribian su nombre de manera precisa, y esta era la tarea solicitada en las dos actividades. Sin embargo, la gráfica también muestra un aumento en la presencia de esta dimensión en el aula, situación que se da por las tareas que la docente titular propone, entre las que se encuentran recortar, escribir el nombre de manera constante, dibujar y pintar con colores, acuarelas, entre otras.

La tarea 9 era la única centrada de manera precisa en el trabajo de patrones por medio de la expresión corporal, y fue donde se presentó el mayor indice de la dimensión biológica. Este comportamiento era previsible dado que, según se ha observado, los niños tienen control segmentado de su cuerpo, 
entonces, de antemano, se esperaba que realizaran las actividades propuestas sin dificultad.

Las actividades que buscaban potenciar el desarrollo de la dimensión biológica se centraron en fomentar capacidades relacionadas con la grafía o con los movimientos del cuerpo, razón por la cual se reconoce que el análisis de esta dimensión es parcial, sin desconocer que acciones como escribir, calcar, reproducir una imagen o una letra y seguir un movimiento físico, entre otras se relacionan con otras dimensiones del niño.

\section{Comunicar}

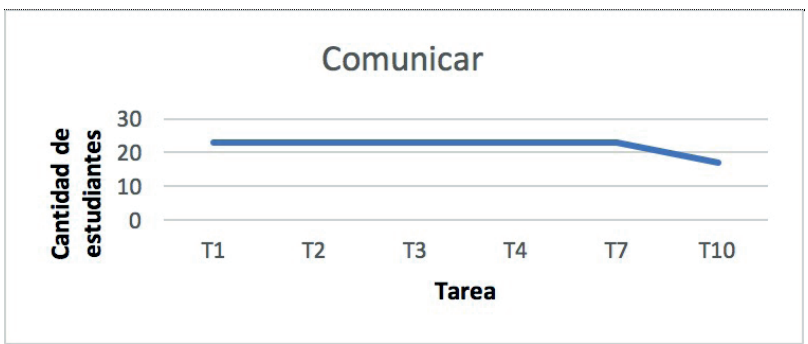

Figura 7. Alcance del proceso de comunicar en la realización de las tareas

Fuente: elaboración propia.

La figura 7 muestra que el trabajo en la clase de matemáticas puede aportar al desarrollo de habilidades comunicativas, lo que da fuerza a la idea de que el área de lenguas no es la única responsable de esta empresa (Valderrama, 2000). De las seis tareas que se proponian potenciar el proceso de comunicar, cinco lograron el objetivo con la totalidad de los estudiantes. Cabe resaltar que para los niños no significa ningún obstáculo no saber escribir en la forma convencional, dado que en todas las actividades que se les solicitaba comunicar una idea hicieron uso de letras, números y dibujos, lo cual mostró su capacidad comunicativa.

La tarea 10 pedía a los niños inventar una secuencia del estado del tiempo para el mes de mayo, lo que implicaba, además de valerse de elementos simbólicos, tener una idea sobre lo que significa un patrón o secuencia. Esta última característica dificultó que algunos estudiantes comunicaran la idea de manera acertada, por lo que hicieron representaciones que no tenían relación con la tarea solicitada.
Finalizados los dos tipos de análisis, se elaboró una gráfica que relaciona el alcance de las tareas en las tres dimensiones de manera simultánea (Véase figura 8).

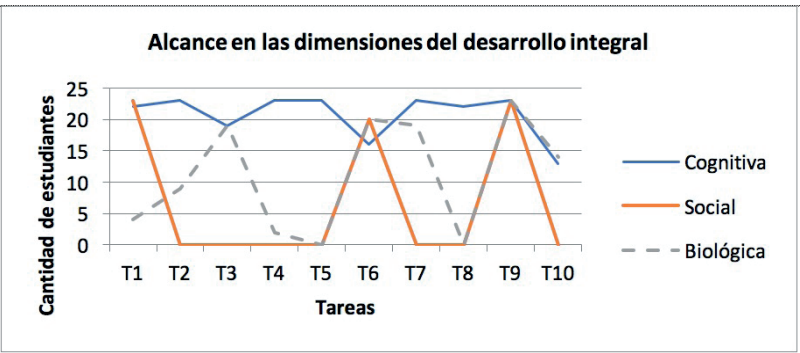

Figura 8. Alcance de las actividades en las dimensiones del desarrollo integral

Fuente: elaboración propia.

La tarea 1 implicaba que el niño comunicara una idea, lo que atiende a la dimensión social, pero para ello se hacian necesarias habilidades cognitivas como escoger los símbolos más adecuados para lograr una representación precisa. Como lo muestra la gráfica, esta tarea integró dos dimensiones del desarrollo integral, junto con el proceso de representar del pensamiento matemático.

La capacidad del niño para escribir no es una habilidad motriz únicamente, en ella intervienen la toma de decisiones, el hacer conexiones con situaciones pasadas y la memorización. Por ello, para la tarea 3 se observa en la gráfica una relación entre la dimensión cognitiva y biológica, mientras también se hace evidente un proceso asociado al desarrollo del pensamiento matemático.

En la tarea 6 se necesitaban las competencias comunicativas que, como ya se ha dicho, están relacionadas con la dimensión cognitiva, por tanto en la gráfica se observa la relación que se presentó al llevar a cabo esta tarea.

Al buscar potenciar el desarrollo integral, la tarea 9 resultó ser la actividad más integradora dado que los niños debian escuchar y proponer ideas, poner en juego la expresión corporal y habilidades cognitivas para la toma de decisiones en relación con los movimientos por realizar. Esto se traduce en la movilización de las tres dimensiones de manera simultánea y, adicionalmente, se presentó un alto índice de estudiantes que alcanzaron los logros correspondientes. 
Por último, al hacer un cruce entre dimensiones y procesos, es evidente la presencia de la dimensión cognitiva asociada a variados procesos, como era de esperarse, pero es satisfactorio observar la presencia de otras dimensiones como la biológica, así como las relaciones entre procesos y dimensiones, por ejemplo: comunicar, resolver problemas y representar con la dimensión biológica y, claro, entre procesos entre sí, como se muestra en la figura 9.

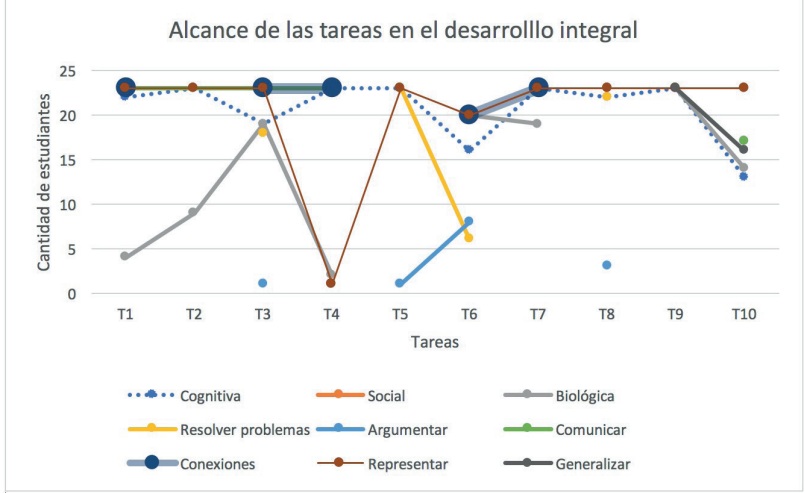

Figura 9. Alcance de las tareas en el desarrollo integral

Fuente: elaboración propia.

Esta gráfica ratificó una idea inicial de este proyecto: potenciar el Pensamiento Matemático contribuye al desarrollo integral del niño que es el objetivo central de los programas de educación, y en particular la educación inicial. Además, hace evidente que no es posible trabajar dimensiones o procesos de manera disjunta, pues para llevar a cabo las actividades acá propuestas los niños expusieron diversos elementos de manera simultánea.

\section{Conclusiones}

Intentar aportar a las tres dimensiones del desarrollo integral, y a los seis procesos del pensamiento matemático establecidos en todas las tareas, resultó un objetivo muy ambicioso que solo se logró de manera general, ya que el análisis por dimensiones/procesos mostró que varios de estos fueron tratados de manera limitada.

La representación del mundo real fue uno de los aspectos más abordados en la realización de las tareas, en actividades como dibujar los elementos de un tesoro o enviar un mensaje. Esta última resultó enriquecedora dado que los niños no saben escribir de manera convencional, por lo que generalmente recurren a otros medios para comunicar una idea, como dibujar la situación o representarla mediante símbolos que ya conocen, por ejemplo algunos numerales.

La interpretación de símbolos que lograban los niños en las tareas que tenían el objetivo de potenciar el proceso de comunicación resultan ser el inicio del trabajo algebraico que no es más que el lenguaje de las matemáticas. Este tipo de tareas cambian los roles tradicionales de los agentes involucrados en el proceso de comunicación en el aula: del docente como emisor, el saber como mensaje y del alumno como receptor pasivo (Valderrama, 2000).

Los niños usaron distintos medios de comunicación cuando de expresar ideas se trataba, entre estos se encuentran el uso de la letra inicial de las palabras que desean usar, dibujos, números, colores y la comunicación oral. En este último caso los estudiantes piden la palabra para expresar sus ideas mientras los demás escuchan.

Durante el desarrollo de las tareas propuestas se evidenció que los niños comprenden el problema presentado, dada la instrucción empiezan a trabajar en una estrategia de solución y en uno de los casos se lanzaron a plantear hipótesis sobre la ubicación del tesoro (tarea 6), a partir de un mapa en el que no había información suficiente.

Este ejercicio de investigación reveló la capacidad de los niños para conectar lo que está sucediendo con experiencias pasadas, dado que, sin importar el tema que se propusiera en el aula, ellos hacian aportes relacionados con situaciones que han vivido o han visto en la televisión.

En ocasiones, los niños debían llevar las ideas que tenían en un plano mental a un plano externo y en otras situaciones debian interpretar la representación dada. En la mayoría de los casos estas situaciones se dieron sin problemas, por lo que se concluye que, en general, los niños hacen traducciones entre representaciones mentales y externas. Por otro lado, las tareas movilizaban distintos medios para representar, por ejemplo, al pedir al niño que realice un doblez en el papel de determinada manera se hizo de forma enactiva, en la mayoría de casos la clase se valía de representaciones icónicas para expresar una idea. 
Las tareas enfocadas al proceso de generalizar tuvieron una respuesta positiva por parte de la clase puesto que identificaron el patrón y lo siguieron sin dificultad. Una vez identificado el patrón se pedía a la clase que inventara uno nuevo, y en la mayoría de los casos los niños crearon un núcleo para la secuencia que deseaban construir.

La conclusión más importante de este ejercicio investigativo es reconocer que el desarrollo de procesos matemáticos no está desligado del desarrollo de las diferentes dimensiones de los niños, sin embargo en ocasiones se desconectan de la enseñanza. La experiencia reportada mostró que es factible poner en práctica el diseño de actividades que conscientemente busquen potenciar uno de estos aspectos, y con ello contribuir a la formación integral de los niños.

\section{Referencias}

Chamorro, M. C. (2003). Didáctica de las matemáticas para primaria. Pearson Educación.

Chamorro, M. C. (2005). Didáctica de las matemáticas para educación infantil. Pearson Educación.

Congreso de Colombia. (8 de febrero de 1994). Ley General de Educación. [Ley 115 de 1994]. D0:41.214.

Duque, K., (2018). Tareas matemáticas para potenciar el desarrollo integral del niño en preescolar. Universidad Pedagógica Nacional. http://repositorio.pedagogica.edu.co/ handle/20.500.12209/9528

Garcia, G. y Salazar, C. (2009). La resolución de problemas de matemáticas: entre la esperanza de cambios en los ambientes de aprendizaje y como herramienta en el control centralizado del currículo. Nodos y Nudos, 3 (27), 70-79.

Ministerio de Educación Nacional, MEN. (1998). Serie Lineamientos curriculares. Preescolar. Autor.

Ministerio de Educación Nacional, MEN. (2006). Estándares básicos de competencias en matemáticas. Autor.

Ministerio de Educación Nacional, MEN. (2017). Derechos Básicos de Aprendizaje, Transición. Autor.
National Council of Teachers of Mathematics (Ed.). (2000). Principles and standards for school mathematics (Vol. 1). National Council of Teachers of Mathematics.

Secretaría de Educación del Distrito. (2013). Lineamiento pedagógico y curricular para la educación inicial en el Distrito. Autor.

Ordoñez, L. A. (2009). La comunicación en clase de matemáticas. Nodos y Nudos, 3 (26), 58-67.

Programme for International Student Assessment. (2012). Marco de Matemáticas. http://www.oecd.org/pisa/test/

Unicef. (2001). Estado Mundial de la Infancia 2001: Primera infancia (Vol. 2001). Unicef.

Valderrama, C. E. (2000). Comunicación-educación: un nuevo escenario. Nodos y Nudos, 2 (8). https://doi. org/10.17227/01224328.1033 


\section{Diálogo del conocimiento}

El desarrollo del pensamiento matemático es de suma importancia en las edades tempranas, pues es alli donde se desencadenan los aprendizajes más significativos, y como lo plantea María Montessori, los niños tienen "mentes absorbentes". Por ello, tal como lo declaran las autoras del artículo, la planeación, gestión y evaluación de actividades potentes para detonar el desarrollo de ese pensamiento requiere de una intencionalidad clara por parte del profesor (educador matemático, educador infantil y/o de la básica primaria), para que a su vez permita aportar a la integralidad del sujeto que se educa.

Este tipo de investigaciones evidencian la posibilidad de descentrar la enseñanza y el aprendizaje de las matemáticas de los objetos fijos e inmodificables, y permiten caminar hacia un cambio en la concepción de la educación matemática.

Elizabeth Torres Puentes Profesora Facultad de Educación - Universidad Pedagógica Nacional 


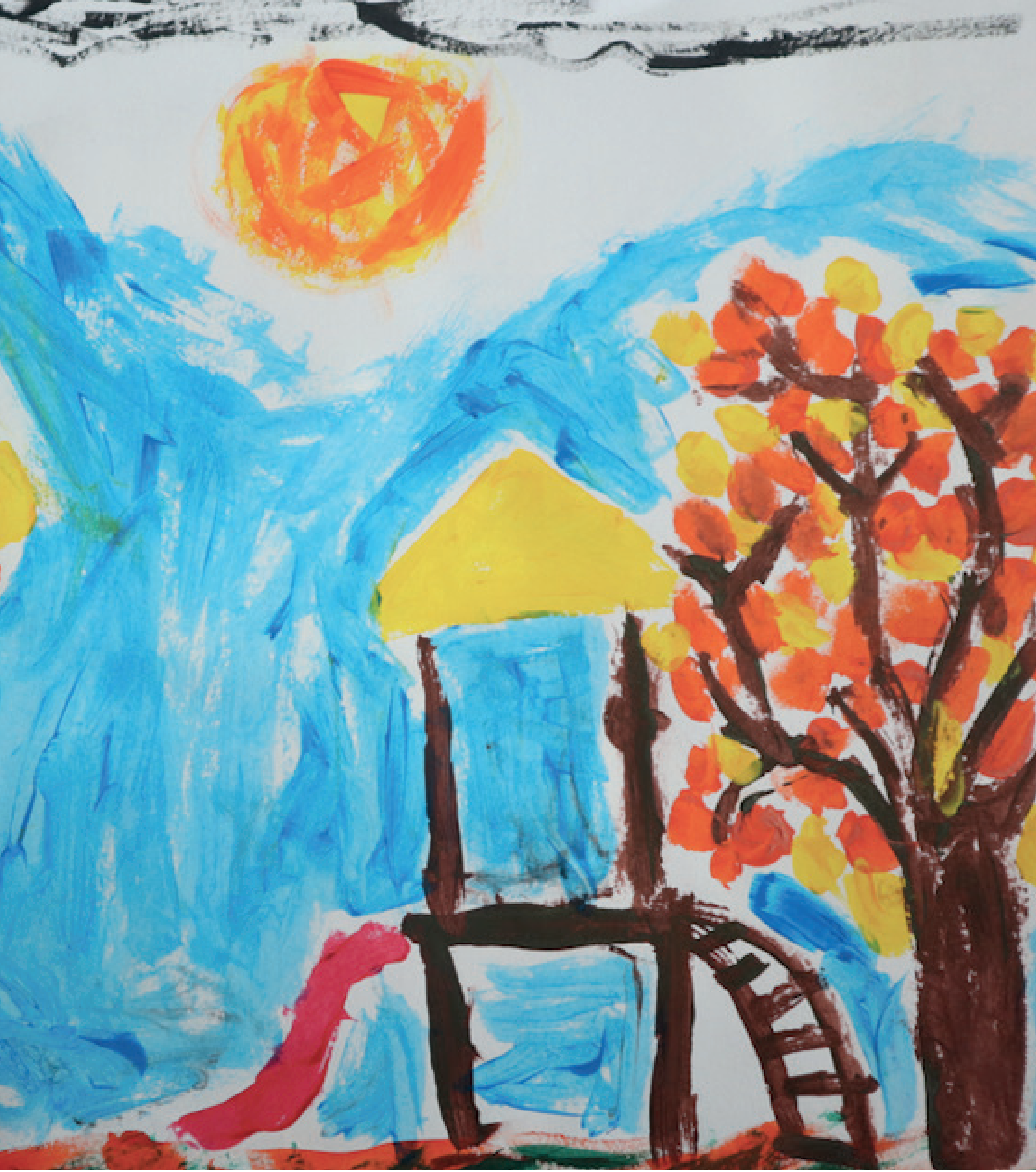

